\title{
The Radio Frequency Source Position Finder Based on The Triangle-Centroid-Algorithm
}

\author{
S. Kristiyana ${ }^{1}$, A. Susanto ${ }^{2}$, Sunarno $^{3}$, R. Hidayat ${ }^{4}$
}

\begin{abstract}
Radio Direction Finder (RDF) had been used as a part of the system in order to determine the direction of the Radio Frequency (RF) source. This research had used three different RDF stations without any mobile human operator. The direction data were collected from the each RDF station, it would be calculated using the triangle-centroid-algorithm. Therefore, the RF source position had been automatically determined. This experiment result of the RF source imaginary was compared with the real RF source at about 3.2 meters for latitude, and 3.3 meters for longitude. The results of this method and experiment are very useful for scientist and engineers in application of RF engineering.
\end{abstract}

Keywords- Radio Frequency, Radio Direction Finder, Triangle-Centroid-Algorithm

\section{INTRODUCTION}

The Radio Direction Finder (RDF) is the activity to find the signal or noise source by tracking the direction using the portable receiver and directional antenna as well. RDF activities are usually done by the amateur radio. The amateur radio are proficient in RDF activity in order to determine the signals of the illegal jammers and malfunctioning transmitters which have noise (interference) [1]. During a national amateur radio contest, there are theoretical findings. The results were originally published by the authors in amateur radio magazines [2] - [4] such as a professional radio direction finder (RDF)-type Rohde \& Schwarz (R\&S) DDF0xM which was used to NVIS Elevation Angle Measurements. Near Vertical Incident Skywave (NVIS) radio wave propagation uses the ionosphere as a reflector, on frequencies ranging from approximately 3 to $10 \mathrm{MHz}$ [5]. NVIS propagation is also used on a daily basis in developing countries, in areas where telecommunication networks are unreliable or nonexistent, providing essential telecommunication such as voice and data communication between small business offices, healthcare facilities, and even banking facilities [6].

${ }^{1}$ Doctoral Student, Dept. of Electrical Engineering and Information Technology, Faculty of Engineering, Universitas Gadjah Mada, Jln. Grafika No. 2, Yogyakarta, 55281, INDONESIA (e-mail: samuel.kristiyana@mail.ugm.ac.id)

${ }^{2,4}$ Lecturer, Dept. of Electrical Engineering and Information Technology, Faculty of Engineering, Universitas Gadjah Mada, Jln. Grafika No. 2, Yogyakarta, 55281, INDONESIA (e-mail: adhisusanto@ugm.ac.id,risanuri@ugm.ac.id)

${ }^{3}$ Lecturer, Dept. of Nuclear Engineering and Engineering Physics, Faculty of Engineering, Universitas Gadjah Mada, Jln. Grafika No. 2, Yogyakarta, 55281, INDONESIA (e-mail: sunarno@ugm.ac.id)
In this research, RDF had been used as a part of the system in order to determine the direction of the Radio Frequency (RF) source [7]. There are some research about high frequency using RDF such as the development and study of a practical spaced-loop RDF for high frequencies [8] and some experiments on conducting screens for a u-type spaced-aerial $\mathrm{RDF}$ in the frequency range 600-1,200 mc/s [9].

RDF was constructed of specific instruments, antennas, and software in order to determine the RF source position [10] [11]. RDF usually utilizes circular or cylindrical arrays. It needs compact and conformal antennas to satisfy the requirements in terms of size, weight and shape, whilst still preserving an impedance bandwidth of at least $100 \%$, a frequency-stable radiation pattern with a reasonable Directivity (as it can improve the accuracy of the DOA estimation and reduce the probability of ambiguity), and a low parasitic coupling between the array elements and the supporting structure. Moreover, antenna elements must feature a stable phase centre for different DOAs [12]

As RDFs generally track narrowband Signals, although a large bandwidth is required, the time domain response of the antenna is not critical. However, a low dispersion profile will be helpful when tracking ultrawide band signals. To increase the mechanical and environmental durability of the antenna array, a cover is necessary. Previous efforts on designing an antenna element dedicated to DOA estimation are based on dipoles and composite dipoles [13], patch antenna arrays with EBG substrates [14], and Switched Parasitic Antennas (SPAs) [15].

Recently, some papers report that the RDF development have been used three methods, i.e., Signal Strength (SS), Time-of-Arrival (TOA), and Direction-of-Arrival (DOA) [16]. The time-of-arrival (TOA) method is commonly method that able to determine the location of object accurately by using at least two stations [17]. The distance is obtained by calculating the arrival time of the object's electromagnetic signal at the stations for the velocity of signals in space is a constant [18] - [20] The time-of arrival differences among remote stations will be calculated, and this will result in several time-difference hyperbolas. The intersection of the hyperbolas will be assumed to be the location of the object.

This paper is focused on the DOA method. The previous researches had reported that the DOA were based on the point of RF source, which the position were assumed are known, i.e., the degree of the angular spread of the RF source [21]. In addition, the DOA detection requires the use of an antenna array with multiple elements [22]. Therefore, the DOA is found ineffective, since it is caused by the requirement of the mobile human operator in order to track the RF source. 


\section{RADIO DIRECTION FINDING (RDF)}

Radio Direction Finding (RDF) systems have many applications in Radio Navigation, Emergency Aid and intelligent operations [23]. The most important information that estimated by the Radio Direction Finding (RDF) system is the Direction Of Arrival (DOA) of the incoming signals. The latest technology such as Software Defined Radio technique make it easy to design as all digital radio receiver which is a flexible open architecture platform with dynamic selection of parameters for individual modules. Recently, a lot of digital radio receiver architectures have been investigated [24].

Due to recent wide spread of wireless technologies, accurate Radio Direction Finders (RDFs) are requested for a broad range of civil and military applications [1]. Such as approach to mitigate the Jitter effect in DOA estimation for the RF signals received by an element Uniform Linear Antenna (ULA) array is proposed. The performance of this method will be assessed in many cases that depend on the characteristics of incoming signals as well as Jitter factor properties [25]. Another research is a variation on the loop antenna topology that suppresses its sensitivity to HP as well as providing advantages in sensitivity and pattern integrity. That present a similar variation of the four-element Adcock array that addresses its sensitivity weakness in electrically small arrays. Both are protected by a provisional patent [26].

Several algorithms are also implemented with the RDF to increase the performance of RDF. For example an implementation of a Radio Direction Finding prototype using three AoA algorithms and MIMO synchronization over a SDR platform, with a non-calibrated two element array. This algorithms were tested in three scenarios with different type of transmitted signals including baseband AM and FM sinusoidal tone and an AM modulated voice signal. The angle was estimated with a mean error below 0.3 [27].

A radio direction finding prototype used an algorithm which is based on angle of arrival estimation techniques. An antenna array designed for this task and the measurement setup are presented. The Spectrum Monitoring Manual of ITU suggests the use of low cost RDF systems for spectrum management activities in developing countries. The low cost feature refers to the use of less accurate, cheaper and portable equipment, allowing the use of several distributed monitoring stations for proper spectrum management in the country [28].

RDF system constructed of several components. Fig. 1 depicts the block diagrams which construct the whole system. Each part of the block diagram of the RDF system are interdependent each other.

The Fig. 2 shows the algorithm of the determining the RF source position. The RF source in specific frequency will be monitored by three RDF stations which had different coordinate. When the RF source transmit the signal, the angle $(\theta)$ will be determined by RDF stations. The each path of the three RDF stations will be a straight line. In ideal or perfect condition, the three lines will pass the same point, i.e., the real RF source position. In fact, the three lines never pass the same point, they construct triangle pattern as depict in Fig. 2. The triangle which is constructed by three lines has a centroid point. This centroid point assumed as a RF source imaginary.

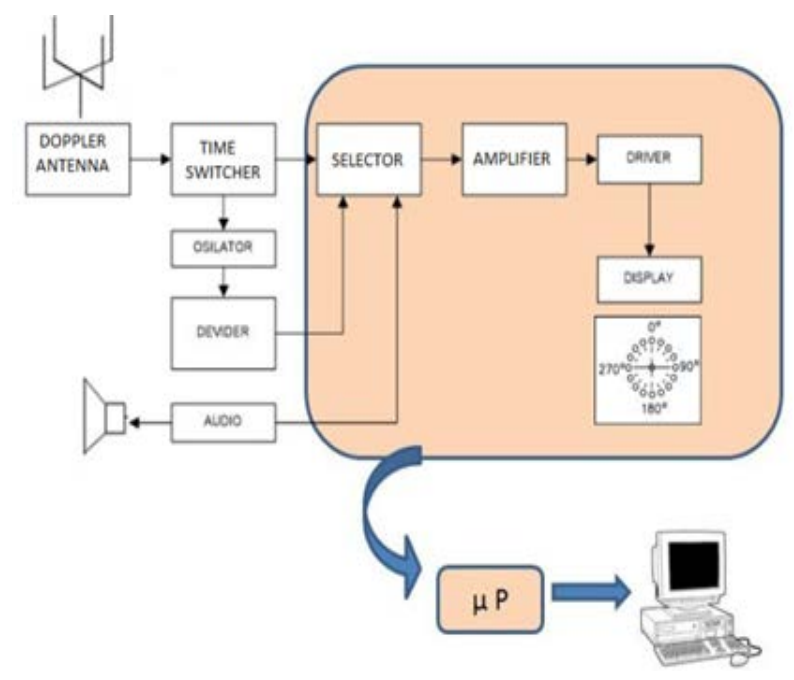

Fig. 1 Block diagram of RDF system [4], [8], [9].

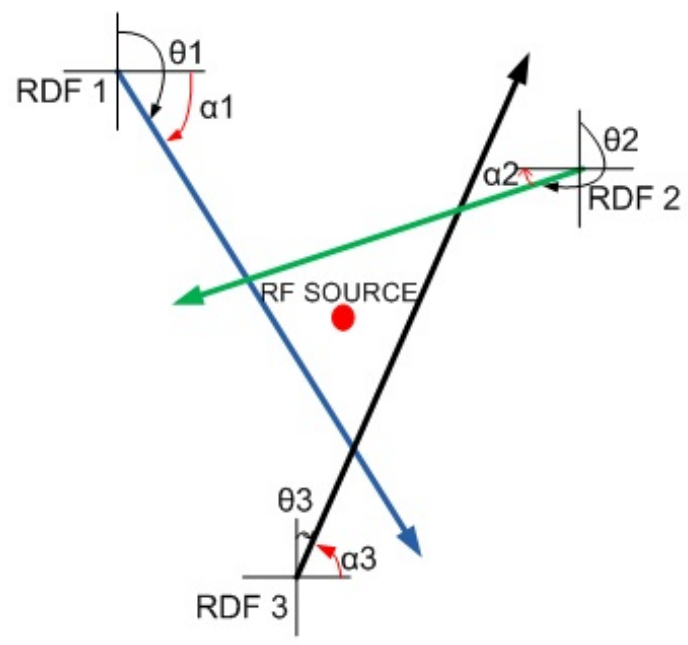

Fig. 2 RDF System Schematic.

The directional angle data of each RDF stations will be calculated to determine the RF source imaginary. In our experiment, the RF source imaginary and the real RF source were compared to determine the accuracy of the system. It will be calculated using the triangle-centroid-algorithm.

Triangle and centroid localization algorithm [29] is an algorithm which combines trilateration and centroid algorithm, but the triangle and centroid localization algorithm is not workable because the assumed condition of three circles intersecting to form an overlapping area cannot be met in practical situations [30]

Fig. 3 shows the schematic of triangle and centroid localization algorithm. Assume that A, B and C are three anchor nodes and D is an unknown node. These four nodes are within communication scope, thus they can communicate directly [31]. $r_{a}, r_{b}$, and $r_{c}$ are distances from A, B and C to D respectively. Draw circles with point $\mathrm{A}, \mathrm{B}$ and $\mathrm{C}$ as centers and $r_{a}, r_{b}$, and $r_{c}$ as radiuses, then an overlapping area is 
formed. The practical unknown node is D. The estimated result of the unknown node is $\mathrm{M}$, which is the centroid of $\triangle E F G$. The first step of practical derivation for this algorithm is getting the coordinates of feature point E, F, G. The other two points have a similar derivation process.

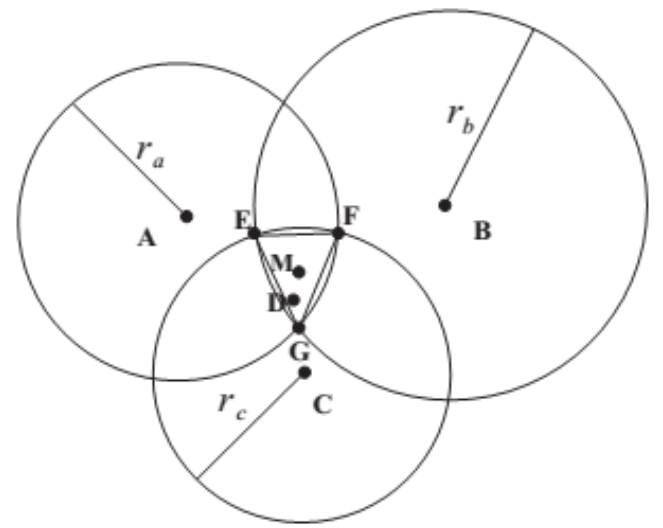

Fig. 3 Schematic of triangle and centroid localization algorithm [30].

\section{TRIANGLE CENTROID ALGORITHM}

The triangle which is constructed by three lines has a centroid point. Each line has specific equation. It assumed that the line was straight line. The three lines constructed a triangle form, therefore, the center of triangle can be determined as an imaginary RF source position. The straight line followed the slope-intercept form of the rectangular coordinate system principle was used to generate each line equation.

Equation (1) depicts the general equation to calculate the slope-intercept form of the rectangular coordinate system, with vertical change divided by horizontal change. In any case, the slope value also call by gradient can be calculated with equation (2). Furthermore, the intercept value, $b$, can be found by equation (3) [32].

$$
y=m x+b
$$

with,

$$
m=\tan \alpha
$$

so,

$$
b=y-x \tan \alpha
$$

In this equation, $\alpha$ generally means $x / y$.

If point RDF1, RDF2, and RDF3 were indexed as $i, j$, and $k$, respectively, furthermore we would have $b_{i}, b_{j}$, and $b_{k}$. The angle point was the crossing point of two lines or when $x_{i}=x_{j}=x_{i j}$ and $y_{i}=y_{j}=y_{i j}$, in case $\mathrm{i}$ cross with $\mathrm{j}$. We would get the value of $x_{i j}$ by substituting (1) for each point; in this case we called (4).

$$
x_{i j}=\frac{c_{j}-c_{i}}{m_{i}-m_{j}}
$$

The variable $x_{i j}$ was used to calculate $y_{i j}$ by using equation (1), (2), and (3) as well. Furthermore, the equation
(4) can be used to calculate the cross-section point of $i$ with $k, x_{i k}$, and $j$ with $k, x_{j k}$, respectively.

The classical centroid localization algorithm is proposed by N. Bulusu and J. Heidemann, the unknown nodes are located by their neighbor anchors which are sending signals that contained their own coordinate, $\left(X_{\text {est }} Y_{\text {est }}\right)$, the centroid calculation algorithm refers to equation (5) [33], [34].

$$
\left(X_{e s t} Y_{e s t}\right)=\left(\frac{X_{1}+X_{2}+\ldots+X_{N}}{N}, \frac{Y_{1}+Y_{2}+\ldots+Y_{N}}{N}\right)
$$

In this research, the classical centroid localization algorithm had been modified in order to determine the RF source imaginary. In this calculation, the centroid point of the triangle is defined by averaging every angle point component, in order to improve the classical centroid localization algorithm.

The centroid in the component of $x$ and component of $y$, are showed in equation (6) and equation (7).

$$
\begin{aligned}
& x_{\text {target }}=\frac{1}{3}\left(x_{i j}+x_{i k}+x_{j k}\right) \\
& y_{\text {target }}=\frac{1}{3}\left(y_{i j}+y_{i k}+y_{j k}\right)
\end{aligned}
$$

The coordinate of the RF source can be determined by the value of $x_{\text {target }}$ and $y_{\text {target }}$. In this case $y_{\text {target }}$ was latitude and $x_{\text {target }}$ was longitude in the Global Positioning System (GPS) mode. Global positioning system (GPS) is a spacebased radio navigation system owned by the United States government and operated by the United States Air Force. It is a global navigation satellite system that provides geolocation and time information to a GPS receiver anywhere on or near the Earth where there is an unobstructed line of sight to four or more GPS satellites. In this research, GPS was used to determine the coordinate of the object's location.

\section{IMPLEMENTATION}

The code was created in order to implement the algorithm and calculation as well. The code requires some parameters of the each RDF source. Each RDF source has three parameters, i.e., latitude, longitude, and the angle in azimuth $(\theta)$.

The code produces some parameters, for example: latitude and longitude from two RDF points, intercept of two lines, and gradient of two lines. The main parameter which is very important of this code is the coordinate of the RF source imaginary. In this research, the RF source imaginary was compared with the real RF source position. Moreover, the accuracy of the system can be defined as well.

Fig. 4 shows the flowchart of the code in order to determine the coordinate of the RF source imaginary. Based on the code results, the coordinate of the RF source imaginary can be projected in Google Map open source engine for the sake of user friendly visualization.

\section{SETUP AND RESUlt}

Three RDF stations were placed in separated location. The position of the RDF stations were set up already. Table I shows the coordinates of the RDF stations. 


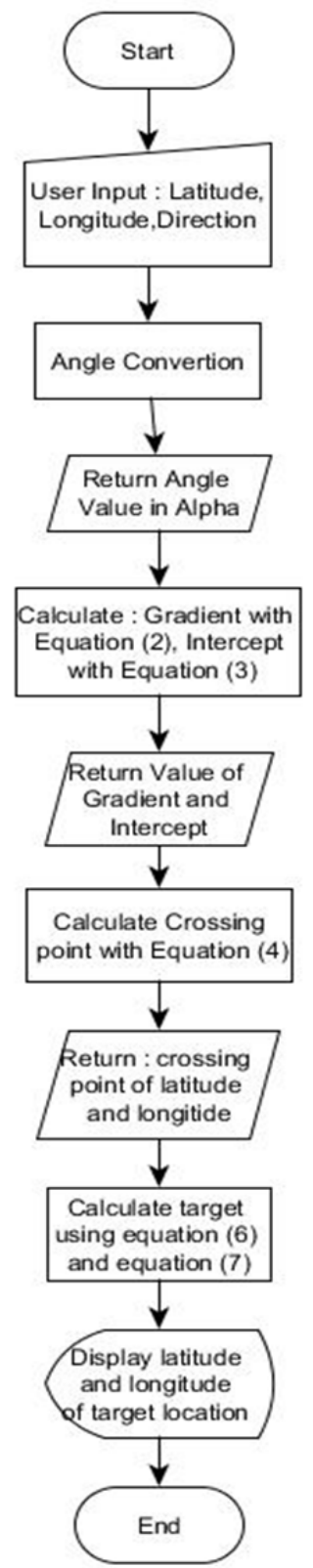

Fig. 4 Flowchart of the code based on the Triangle-Centroid Algorithm.

TABLE I

THE COORDINATE OF RDF STATIONS

\begin{tabular}{|c|c|c|}
\hline Direction Finder Location & Longitude $\mathbf{(}^{\mathbf{}}$ ) & Latitude $\mathbf{(}^{\mathbf{}}$ ) \\
\hline RDF 1 & 110.1794 & -7.647167 \\
\hline RDF 2 & 110.4633 & -7.597861 \\
\hline RDF 3 & 110.5518 & -7.839806 \\
\hline
\end{tabular}

The RDF stations were oriented to the RF source direction. Fig. 4 shows the position of RF source.

Fig. 5 shows that the longitude value of the RF source imaginary is compared with the real RF source position. The direct measurement by Global Positioning System (GPS) was measured 100 repetitions and shows the result not consistently, but the output program always has the result consistently.

\section{RF LONGITUDE GRAPH}

$\downarrow$ Direct Measurement $\quad$ Output Program

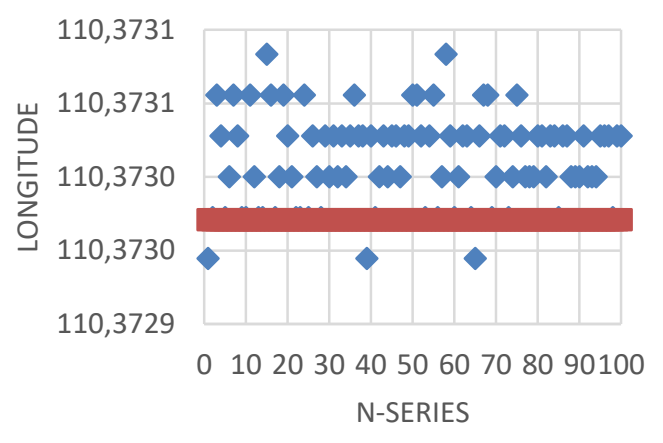

Fig. 5 Comparison graphic between latitude value at RF source location using direct measurement and output program.

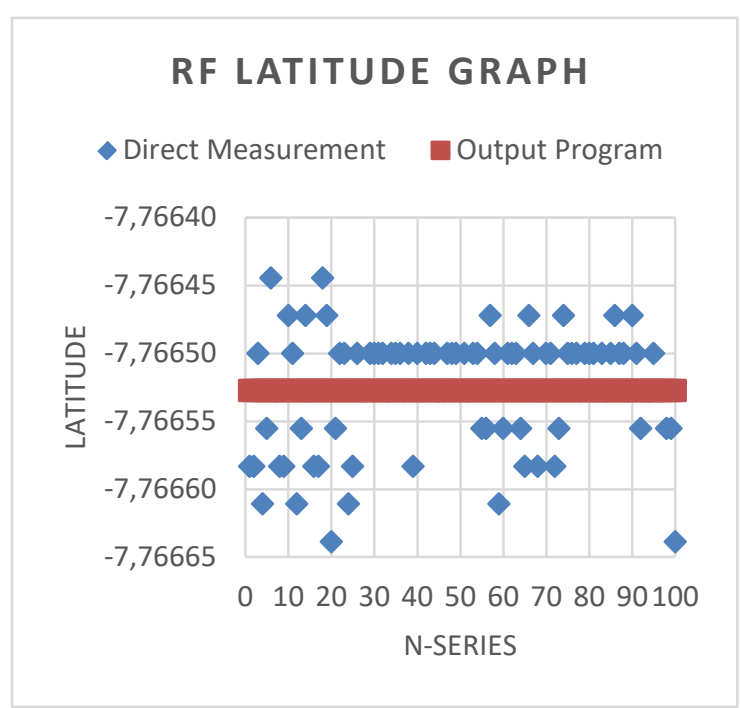

Fig. 6 Comparison graphic between latitude value at RF source location using direct measurement and output program.

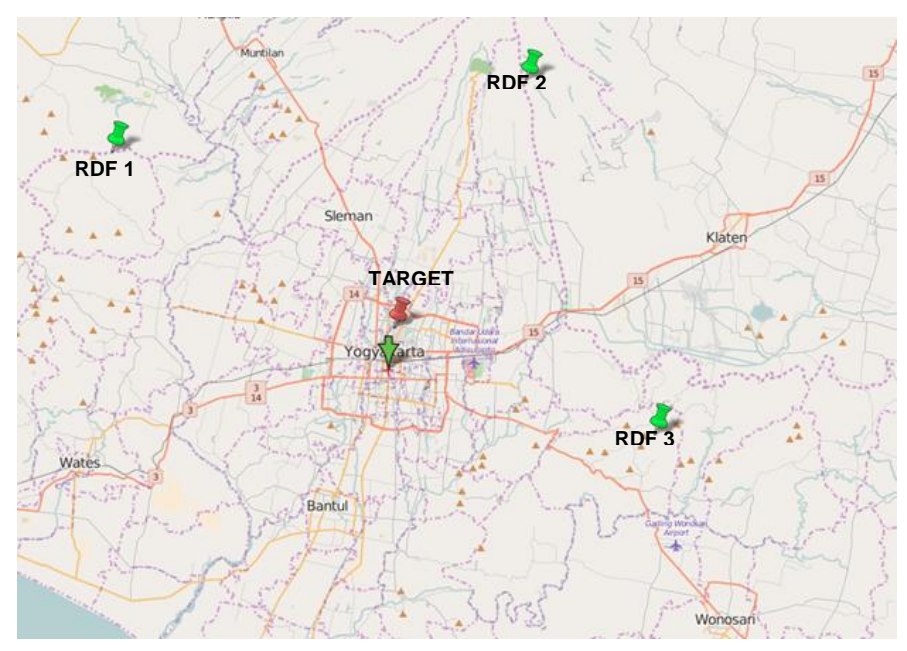

Fig. 7 Visualized RF source location from code program that integrated with Google Map. 
Fig. 6 shows that the latitude value of the RF source imaginary was compared with the real RF source position. The direct measurement by GPS was measured 100 repetitions and shows the result not consistently, but the output program always has the result consistently.

The Fig. 7 shows the RF source imaginary that calculated using the triangle-centroid-algorithm. The RF source imaginary is indicated at latitude $-7.766527^{\circ}$, longitude $110.3730^{\circ}$ meanwhile the real RF source position at latitude $7.766556^{\circ}$, longitude $110.3730^{\circ}$. The experiment result shows that the difference of the latitude and longitude from The RF source imaginary compared with the real RF source is 3.2 meters for latitude, whereas 3.3 meters for longitude.

\section{CONCLUSION}

This research uses three different RDF stations without any mobile human operator. The direction data are collected from the each RDF stations; it is then calculated using the trianglecentroid-algorithm. Therefore, the RF source position is automatically determined.

In this research, the RF source imaginary is calculated using the triangle-centroid-algorithm. This experiment result the RF source imaginary compared with the real RF source is about 3.2 meters for latitude, and 3.3 meters for longitude.

\section{ACKNOWLEDGEMENTS}

The research for this paper was supported by the Sensor and System Telecontrol Laboratory, Digital System Laboratory, and Electronic Instrumentation System Laboratory, Universitas Gadjah Mada.

\section{REFERENCES}

[1] H. W. Silver, The ARRL Handbook for Radio Communications, Newington: ARRL , 2014.

[2] B. A. Witvliet, E. V. Maanen, G. J. Petersen and A. J. Westenberg, "Impact of a Solar X-Flare on NVIS Propagation," IEEE AntEnnAs \& ProPAgAtIon MAgAzInE, vol. 10.1109, no. december, pp. 29-36, 2016.

[3] B. A. Witvliet, G. J. Laanstra, E. V. Maanen and R. M. Alsina-pagès, "A Transportable Hybrid Antenna-Transmitter System for the Generation of Elliptically Polarized Waves for NVIS Propagation Research," in Antennas and Propagation (EuCAP), 2016 10th European Conference on, Davos, Switzerland, 2016.

[4] B. A. Witvliet, E. v. Maanen, A. J. Westenberg and G. Visser, "Elevation angle measurements for NVIS propagation," RadCom, vol. 81, pp. 76-79, 2005.

[5] B. A. Witvliet, E. v. Maanen, G. J. Petersen, A. J. Westenberg, M. J. Bentum, C. H. Slump and R. Schiphorst, "Near Vertical Incidence Skywave Propagation: Elevation Angles and Optimum Antenna Height for Horizontal Dipole Antennas," IEEE Antennas and Propagation Magazine, Vol. 57, No. 1, February 2015, vol. 57, no. February, pp. 129-146, 2015.

[6] M. A. Wallace, "HF radio in Southwest Asia," IEEE Commun. Mag, vol. 30, no. Jan, p. 58-61, 1992..

[7] M. C. E. Stieber, "Radio direction finding network receiver design for low-cost public service applications," California Polytechnic State University, San Luis Obispo, 2012.

[8] M. A. M. W. Ross, "The development and study of a practical spaced-loop radio direction-finder for high frequencies," RADIO SECTION, 1946.

[9] B. P. A. M. R. R. Pearce, "Some experiments on conducting screens for a u-type spaced-aerial radio direction-finder in the frequency range 600-1 $200 \mathrm{Mc} / \mathrm{s}, "$ in Radio Section paper (official communication from the National Physical Laboratory), 1947.

[10] P. Denisowski, an Introduction to Radio Direction Finding Methodologies, ROHDE\&SCHWARZ, 2015.

[11] S. Hawibowo, Sunarno, M. M. Waruwu and R. Wijaya, "Development of Data Communication System for Virtual Hospital Scheme between Public Health Centers at Remote Areas and Regency Public Hospital Based on Radio Frequency Communication," Journal of Theoretical and Applied Information Technology, vol. 84, no. 2, pp. 294-297, 2016.

[12] I. Liberal, D. Caratelli, A. Yarovoy, R. Cicchetti and M. Russo, "Conformal Butterfly Antennas for Ultra-Wideband Radio Direction Finding Applications," in Proceedings of the 40th European Microwave Conference, Paris, France, 2010.

[13] J. Choi, J. So, C. Park and S. Oh, "Active composite dipole antenna for direction finding array antenna applications," in IEEE APS/URSI Conference, pp 1153-1156; 2006.

[14] A. Hirata, "Accuracy compensation in direction finding using patch antenna array with EBG structure," IEEE Antennas and Wireless Propagation Letters, vol. 5, no. Dec, pp. 1-3, 2006.

[15] T. Svantesson and M. Wennstrom, "High-resolution direction finding using a switched parasitic antenna," Proceedings of the 11th IEEE Signal Processing Workshop on Statistical Signal Processing, pp. 508-511, 2011.

[16] Creative Commons Attribution-Share Alike 3.0, [Online]. Available: //creativecommons.org/licenses/by-sa/3.0/.

[17] N. P, M. I and C. T. Spatio, "Temporal analysis of lightning activity over Greece," Atmos Res , 2014:207-17144 2014:207-17..

[18] Fagan and R. M. D, "Intelligent time of arrival estimation," in IEEE forum on integrated and sustainable transportation system (FISTS), 2011.

[19] e. a. Zeng Q, "The application of lightning forecasting based on surface electrostatic field observations and radar data," J Electrostat, vol. 71(1), pp. 6-13, 2013.

[20] e. a. Yoshida S, "Initial results of LF sensor network for lightning observation and characteristics of lightning emission in LF band," $J$ Geophys Res Atmos, vol. 119(21), pp. 12,034-12,051, 2014.

[21] C. Wen, G. Shi and X. Xie, "Estimation of Directions of Arrival of Multiple Distributed Sources for Nested Array," IEEE Signal Processing Letters , vol. 21, no. 9, Sept, pp. 1111 - 1114, 2014.

[22] A. Lin and H. Ling, "Doppler and Direction-of-Arrival (DDOA) Radar for Multiple-Mover Sensing," IEEE Transactions on Aerospace and Electronic Systems, pp. 1496-1509, 2007.

[23] K. VonEhr, S. Hilaski, B. E. Dunne and J. Ward, "Software Defined Radio for Direction-Finding in UAV Wildlife Tracking," IEEE International Conference on Electro Information Technology (EIT), pp. 0464-0469, 2016.

[24] V. Y. Vu and A. B. DelaY, "New receiver architecture for localisation system," International Symposium on Intelligent Signal Processing and Communication Systems (ISPACS), vol. 06, pp. 879882, 2016 .

[25] H. T. Thanh, T. H. Son, P. T. Minh, D. T. Tuan and V. V. Yem, " Jitter mitigation in radio direction finding system," 2016 IEEE Sixth International Conference on Communications and Electronics (ICCE), vol. 10, pp. 253-257, 2016.

[26] C. A. W. Vale, "Variations on Loop and Adcock Array Topologies for improved Radio Direction Finding Performance," in 2012 IEEEAPS Topical Conference on Antennas and Propagation in Wireless Communications (APWC), 2012.

[27] A. D. Redondo, T. Sanchez, C. Gomez, L. Betancur and R. C. Hincapie, "MIMO SDR-based Implementation of AoA Algorithms for Radio Direction Finding in Spectrum Sensing Activities," in IEEE Colombian Conference on Communication and Computing (IEEE COLCOM 2015), 2015. 
[28] A. Gorcin, H. Celebi, K. A. Qaraqe and H. Arslan, "A Framework on Wideband Sensing and Direction Finding for Location Aware Public Safety Cognitive Radio," in WAMICON 2011 Conference Proceedings, 2011.

[29] W. L. a. C. Chen, "RSSI-based Triangle and Centroid Location in Wireless Sensor Network," Modern Electronics Technique, vol. 32(2), pp. 45-48, 2009.

[30] Y. Shang, Z. Liu, J. Wang and X. Xiao, "Triangle and centroid localization algorithm based on distance compensation," IET International Conference on Information Science and Control Engineering 2012 (ICISCE 2012), pp. 1-4, 2012.
[31] J. Wang, Z. Wang, L. Zhang and F. Shi, "An improved range-based localization algorithm in wireless sensor network," Proceedings of 2011 4th International Conference on Biomedical Engineering and Informatics, no. Oct, pp. 2157-2161, 2011.

[32] D. Varberg, E. Purcell and S. Rigdon, Calculus Ninth Edition, Pearson, 2007.

[33] L. Jie, W. Qing, Z. Hu and S. Jun-de, "An Ellipse-Centroid Localization Algorithm in Wireless Sensor Networks," 2009.

[34] M. Chen and H. Liu, "Enhance Performance of Centroid Algorithm in Wireless Sensor Networks," in Fourth International Conference on Computational and Information Sciences, 2012. 